\title{
Modeling flexible amphiphilic bilayers: A solvent-free off-lattice Monte Carlo study
}

\author{
Zun-Jing Wang ${ }^{\mathrm{a})}$ and Daan Frenkel \\ Fundamenteel Onderzoek der Materie (FOM), Institute for Atomic and Molecular Physics, Kruislaan 407, \\ 1098 SJ Amsterdam, The Netherlands
}

(Received 16 February 2005; accepted 13 April 2005; published online 22 June 2005)

\begin{abstract}
We present a simple, implicit-solvent model for fluid bilayer membranes. The model was designed to reproduce the elastic properties of real bilayer membranes. For this model, we observed the solid-fluid transition and studied the in-plane diffusivity of the fluid phase. As a test, we compute the elastic-bending and area-compressing moduli of fluid bilayer membranes. We find that the computed elastic properties are consistent with the available experimental data. () 2005 American Institute of Physics. [DOI: 10.1063/1.1927509]
\end{abstract}

\section{INTRODUCTION}

Nature uses lipid bilayer membranes to encapsulate and protect both cells and subcellular organelles. ${ }^{1}$ The mechanical properties of these membranes are closely related to their biological functions (see, e.g., Ref. 2). For this reason, there is much interest in developing reliable computational models for bilayer membranes. In some cases, it is possible to use fully atomistic models. Unfortunately, with current computers the applicability of fully atomistic models is mostly limited to studies of a few hundred lipids for a period of a few nanoseconds. ${ }^{3,4}$ In order to bridge the gap between simulations and experiments that probe the structure and dynamics of membranes on longer length and time scales, we are forced to use simplified models, i.e., models that account only for those degrees of freedom that are believed to be most essential for the observed phenomena. The simplest models in this category are not particle based: they include tethered solid membranes, ${ }^{5,6}$ tethered fluid membranes, ${ }^{7,8}$ and continuous elastic sheets. ${ }^{9-11}$ Nonatomistic but particlebased models constitute the next step in coarse-grained $(\mathrm{CG})$ modeling of membranes. In these CG models, groups of atoms within the lipid molecules are replaced by coarsegrained particles. The lipid molecules are then represented as short chains of coarse-grained particles. During the past few years, a large number of such CG models have been developed to study monolayer membranes, bilayer membranes, and vesicles. ${ }^{12-28}$ There are great variations in the CG lipidchain structure, the shape of the coarse-grained monomers, the internal degrees of freedom of the lipids, and the effective interactions between two particles, depending on the membrane properties that one aims to reproduce.

Closest to the fully atomistic models are off-lattice CG models with explicit solvent. ${ }^{12-21}$ While these CG models are better suited for the simulation of large membranes than fully atomistic models, they are still not exactly cheap and this limits their applicability to the study of slow or large-scale phenomena. One way to reduce the computational cost of CG membrane simulations is the use of lattice models. ${ }^{22,23}$

${ }^{a)}$ Electronic mail: wang@amolf.nl
Though computationally cheap, these models tend to produce spurious lattice effects, especially for simulations of the fluid membrane, because they fail to provide a reasonable description of, for instance, small-scale diffusion and molecular tilt. ${ }^{22}$ In what follows, we therefore restrict the discussion to off-lattice models.

In particle-based membrane simulations, a large fraction of the computational cost is related to the simulation of the (many) solvent particles. For this reason, it is attractive to consider models that do not take the solvent into account explicitly-although the effect of the solvent on the membrane structure should of course be incorporated in the model. Solvent-free models have been used to analyze the equilibrium behavior of cell membranes for nearly 30 years. ${ }^{29-33}$ However, in molecular simulations, such models have a much shorter history. Drouffe et al. ${ }^{24}$ performed a solvent-free molecular simulation of a membrane where the solvent-lipid interaction was represented via a densitydependent multibody interaction between lipid molecules. This was an important advance, as the length scale studied in the simulations of Ref. 24 is difficult to reach in simulations with explicit solvent, even with modern computers. A more sophisticated version of this model was subsequently proposed by Noguchi and Takasu. ${ }^{25}$

Recently, Farago ${ }^{26}$ proposed an implicit-solvent model where the lipid molecules are represented by rigid trimers. This model assumed only pairwise additive interactions. Brannigan and Brown ${ }^{27}$ proposed yet another pair-potentialbased solvent-free model, in which the lipid molecule is modeled as a rigid rod that is capable of changing shape. Though these models are computationally very efficient, they have not been particularly successful in reproducing the elastic properties of real lipid membranes. The bending moduli of simulated bilayers are either smaller ${ }^{24,25}$ or much larger ${ }^{26,28}$ than the experimental range. ${ }^{34}$ In addition, as these models represent lipids as rigid chains, they are not sufficient for studying some mechanisms which are very sensitive to the flexibility of the lipid tails, e.g., the free-energy barrier of defect formation in membranes, although they can reproduce some self-assembled or phase-transition phenomena. ${ }^{24-27}$ 


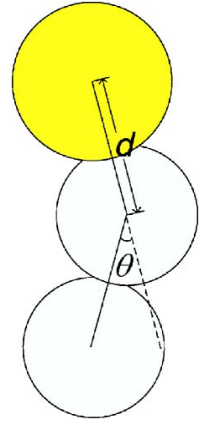

FIG. 1. Illustration of a coarse-grained surfactant molecule which has one hydrophilic bead (solid) and two hydrophobic beads (open).

To remedy these defects, while retaining the computational simplicity of implicit-solvent models, we propose a water-implicit membrane model consisting of flexible, coarse-grained lipids. The remainder of this paper is organized as follows. In Sec. II we describe the simulation model. In Sec. III we present numerical simulations of the phase behavior, fluidity, and membrane elasticity. We compare our results with those of other simulations and with experiment. Section IV contains concluding remarks and we comment on further developments.

\section{SIMULATION METHOD}

\section{A. Potential model}

Each amphiphile molecule is described as a flexible chain with three coarse-grained beads (see Fig. 1). One particle is hydrophilic, the other two are hydrophobic.

The bonds between the beads are represented by a finite extensible nonlinear elastic (FENE) potential ${ }^{13}$

$$
U_{s}(d)=-\frac{1}{2} k_{s} d_{s}^{2} \ln \left\{1-\left[\left(d-d_{0}\right) / d_{s}\right]^{2}\right\} .
$$

The angle-bending potential between two bonds is

$$
U_{b}(\theta)=k_{b}(1-\cos \theta),
$$

where $\theta$ is the angle between subsequent bonds. Beads on different molecules interact via a truncated Lennard-Jones potential:

$$
\begin{aligned}
& U_{t t}(r)=\epsilon\left[\left(\sigma_{t t} / r\right)^{12}-2\left(\sigma_{t t} / r\right)^{6}+U_{c 0}\right], \\
& U_{h h}(r)=\epsilon\left[\left(\sigma_{h h} / r\right)^{12}-2\left(\sigma_{h h} / r\right)^{6}+U_{c 1}\right], \\
& U_{t h}(r)=\epsilon\left[\left(\sigma_{t h} / r\right)^{12}-2\left(\sigma_{t h} / r\right)^{6}+U_{c 1}\right],
\end{aligned}
$$

where $t$ and $h$ represent the tail and head beads, respectively. The cutoffs for potential $U_{t t}, U_{h h}$, and $U_{t h}$ are $2 \sigma_{t t}, \sigma_{h h}$, and $\sigma_{t h}$, respectively, which make attractive interactions exist only between tail beads. $U_{c 0}$ and $U_{c 1}$ (respectively, 0.031 and 1) are the amounts by which the potential is shifted at the cut-off distance.

Water molecules are not taken into account explicitly. We mimic the interaction between the water molecules and amphiphiles with a multibody potential that depends on the local density of the hydrophobic beads

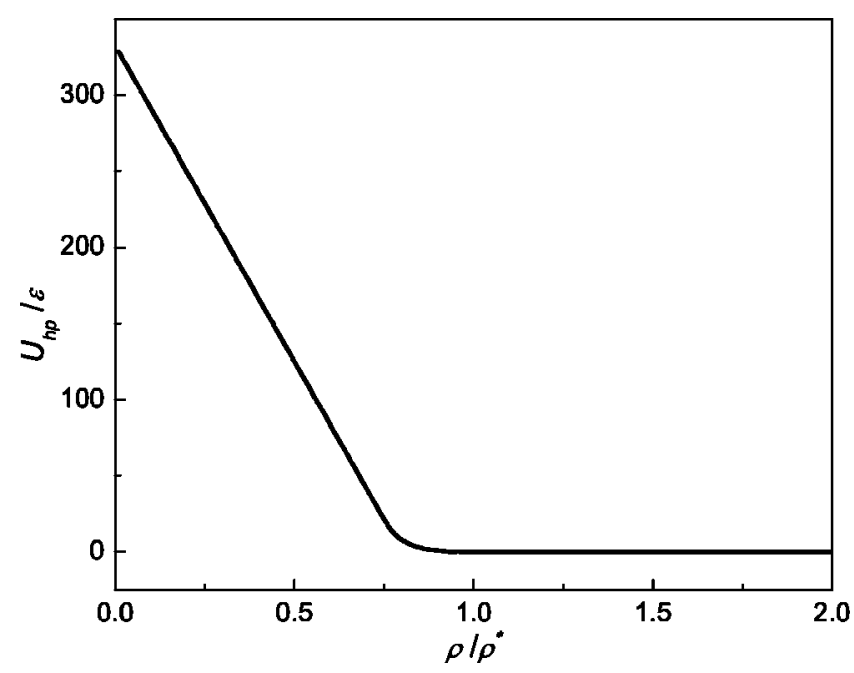

FIG. 2. Dependence of the multibody "hydrophobic" potential on the local density of hydrophobic tail beads (see text).

$$
U_{h p}(\widetilde{\rho})= \begin{cases}\epsilon\left[k_{\tilde{\rho}}(\tilde{\rho}-0.75)+U_{0}\right], & \tilde{\rho}<0.75 \\ \epsilon\left[(1 / \widetilde{\rho})^{12}-2(1 / \widetilde{\rho})^{6}+1\right], & 0.75 \leqslant \tilde{\rho}<1 \\ 0, & \tilde{\rho} \geqslant 1,\end{cases}
$$

where $\tilde{\rho}=\rho / \rho^{*}$. To obtain a potential with a continuous derivative, $k_{\tilde{\rho}}$ and $U_{0}$ are given the values of -415.21 and 21.332, respectively.

This multibody "hydrophobic" potential expresses the increase in free energy due to the contact of water molecules with the hydrocarbon beads of the amphiphiles. As shown in Fig. 2, the critical local density $\rho^{*}$ represents the crossover point below which there is interaction between water molecules and the hydrophobic beads.

In principle, the total potential energy should be rotationally invariant. However, as we consider bilayers in a fixed geometry ( $z$ axis perpendicular to the bilayer), we use a definition of the local density that is optimized for this geometry.

$$
\rho_{i, j}=\sum_{i^{\prime} \neq i, j^{\prime} \neq 1} h\left(\left|\mathbf{r}_{e i, j}-\mathbf{r}_{e i^{\prime}, j^{\prime}}\right|\right),
$$

where $i$ and $i^{\prime}$ represent molecules, $j$ and $j^{\prime}$ represent hydrophobic tail beads, $\mathbf{r}_{e}=x \mathbf{i}+y \mathbf{j}+\alpha_{e} z \mathbf{k}$, and

$$
h\left(r_{e}\right)=\frac{\left(\sigma_{h p} / r_{e}\right)^{12}-2\left(\sigma_{h p} / r_{e}\right)^{6}+1}{\left(\sigma_{h p} / r_{e}\right)^{12}-2\left(\sigma_{h p} / r_{e}\right)^{6}+2},
$$

where the cutoff for $h\left(r_{e}\right)$ is $\sigma_{h p} . h\left(r_{e}\right)$ is approximately the number of hydrophobic particles in the ellipsoid sphere $x^{2}$ $+y^{2}+\alpha_{e}^{2} z^{2}=\sigma_{h p}^{2}$. Note that with this definition of the local density, the many-body potential is related to the choice of the coordinate frame. In general, this would be problematic. However, as long as we consider planar membranes that are periodically continued in the $x y$ plane, say, the symmetry of the system has already been broken.

All the parameters of the potential functions are given in Table I. 
TABLE I. Parameters of the potential functions. $k_{s}$ denotes the spring constant, $d_{s}$ the cutoff, and $d_{0}$ the bond length of the FENE potential [Eq. (1)]. The stiffness constant $k_{b}$ is defined in Eq. (2). The tail diameter $\sigma_{t}$, the head bead diameter $\sigma_{t t}$, and the parameter of interaction between head and tail beads $\sigma_{t h}=0.5\left(\sigma_{t t}+\sigma_{h h}\right)$ are used in the Lennard-Jones (LJ) potentials of Eqs. (3)-(5). $\rho_{t 1}^{*}$ and $\rho_{t 2}^{*}$ denote, respectively, the critical local densities of the first and second tail beads in Eq. (6). $\sigma_{h p}$ and $\alpha_{e}$ are potential parameters that are defined by Eq. (8).

\begin{tabular}{ccccccccccc}
\hline \hline$k_{s} / \epsilon$ & $d_{s} / \sigma$ & $d_{0} / \sigma$ & $k_{b} / \epsilon$ & $\sigma_{t t} / \sigma$ & $\sigma_{h h} / \sigma$ & $\sigma_{t h} / \sigma$ & $\rho_{t 1}^{*}$ & $\rho_{t 2}^{*}$ & $\sigma_{h p} / \sigma$ & $\alpha_{e}$ \\
\hline 100 & 0.2 & 1.0 & 10 & 1 & 1.1 & 1.05 & 10 & 17 & 1.9 & $\sqrt{2}$ \\
\hline \hline
\end{tabular}

\section{B. Statistical ensemble}

We performed Monte Carlo simulations in both the $N V T A_{p}$ and $N V T \Sigma$ ensembles. $N$ is the number of molecules, $V$ is the volume of the simulated system, $T$ is the temperature, $A_{p}$ is the projected area of the bilayer on the $x-y$ plane, and $\Sigma$ is the lateral pressure of the bilayer. For the NVT $\Sigma$ simulations every Monte Carlo sweep includes the following trial moves: (1) $3 \mathrm{~N}$ displacements of a single bead, (2) $\mathrm{N}$ displacements of a single molecule, and (3) a variation of the area of the simulation box at constant total volume. For the area trial move, the coordinates of the head beads are changed, but all intramolecular distances and angles are kept constant. The trial moves are accepted or rejected according to the standard Metropolis criterion with the effective Hamiltonian

$$
H=E-\Sigma A_{p}-N \beta^{-1} \ln \left(A_{p}\right),
$$

where $\beta=k_{B} T$, and $k_{B}$ is Boltzmann's constant. Figure 3 shows the time evolutions of the area and the energy of the membrane during a $N V T \Sigma$ simulation. The figure shows the approach to equilibrium of both the (projected) area and the internal energy of the system.

\section{Simulation details}

Periodic boundary conditions were used in the simulations. The $z$-axis length of the simulation box, which is the direction perpendicular to the membrane, is large enough to exclude interactions between the membrane and its periodic

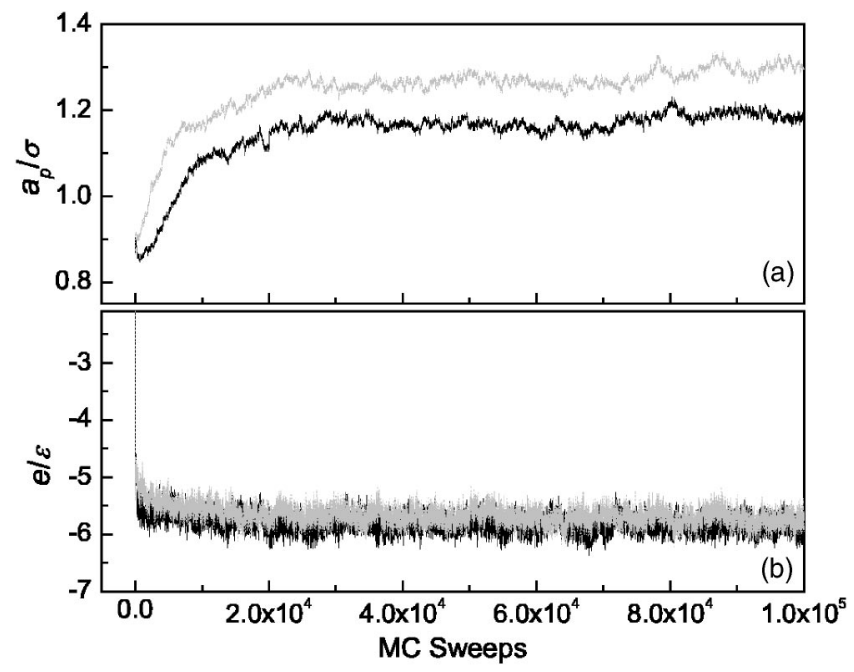

FIG. 3. Temporal evolution of the area (a) and energy (b) per molecule during a Monte Carlo simulation at $k_{B} T / \epsilon=0.8, \quad \Sigma=0$ (dark), and $\Sigma$ $=2 \epsilon / \sigma^{2}$ (light), respectively. images. The system size varied from 200 amphiphilic molecules for simulations of the phase behavior, to 1152 in studies of the area-compressing elasticity and 2040 in simulations of the bending elasticity. In every simulation, the system was equilibrated during $3 \times 10^{5}$ Monte Carlo (MC) sweeps.

In the $N V T A_{p}$ simulations, we either prepared the system as a random [three-dimensional (3D) liquid] state or as a flat, ordered bilayer membrane. When, at $k_{B} T / \epsilon=2.0$, the initial state is a 3D liquid state, we find that the amphiphilic molecules spontaneously form several bilayer membranessometimes with pore defects. When, at the same temperature, the initial state is flat, the equilibrated state is a fluctuating liquid bilayer membrane. In the remainder of this paper, we will always consider bilayer membranes that were formed by equilibrating an ordered, flat membrane.

In what follows, we choose our energy and length scales such that the model parameters correspond to those of typical bilayer membranes at room temperature. To this end, $\epsilon / k_{B}$ roughly has a value in the vicinity of $150 \mathrm{~K}$, while $\sigma$ $\sim 5-8 \AA .{ }^{14}$ With this choice, $k_{B} T / \epsilon=2.0$ corresponds to room temperature, and the length of our model molecules is in the range of 6-24 methyl groups. This covers the relevant range of chain lengths for fatty acids. ${ }^{14}$ As we will see later this model also leads to a reasonable value of area per lipid.

\section{RESULTS AND DISCUSSION}

\section{A. Phase behavior \\ 1. Solid-liquid transition}

As a first step, we observed the solid-liquid transition of a tensionless membrane $(\Sigma=0)$. Figure 4 shows the melting of the solid phase upon increasing the temperature.

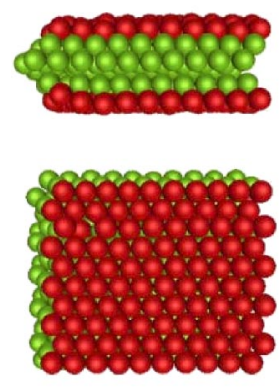

(a)
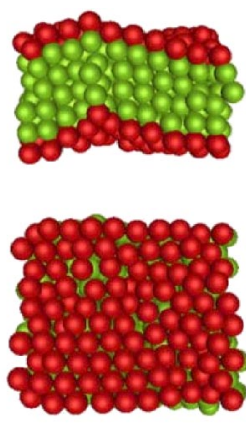

(b)
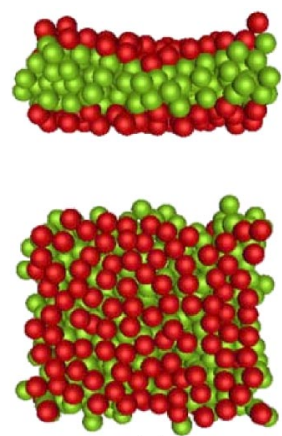

(c)
FIG. 4. Snapshots of the temperature dependence of the structure of a small membrane during a $N V T \Sigma$ simulation at zero lateral pressure. From (a) to (c): $k_{B} T / \epsilon=0.1,0.3,2.0$, which schematically show how the solid membrane melts into liquid with an increase of temperature. 


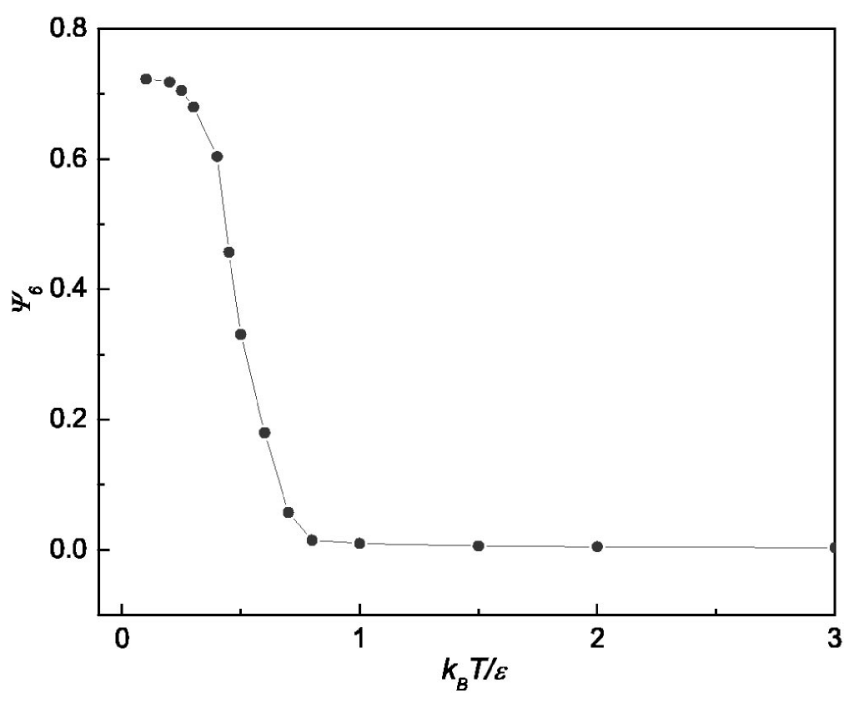

FIG. 5. Temperature dependence of the bond-order parameter of the surfactant head groups at zero lateral pressure.

We use a two-dimensional hexagonal bond-order parameter to quantify the lateral order-disorder transition of a bilayer membrane: ${ }^{28}$

$$
\psi_{6}=\left|\left\langle e^{i 6 \alpha_{i j}}\right\rangle\right|,
$$

where $\alpha_{i j}$ is the angle between $\mathbf{r}_{x y}(i, j)$ and a fixed reference axis in the $x-y$ plane, $\mathbf{r}_{x y}(i, j)$ is the in-plane vector between head beads $i$ and $j$, and the average is over all nearestneighbor pairs $i$ and $j$.

Upon increasing the temperature, the membrane appears to go through a continuous order-disorder transition (see Figs. 4 and 5). The profile of the bond-order parameter indicates that the melting temperature of our model membrane is $k_{B} T / \epsilon<0.5$, which in our units corresponds to $75 \mathrm{~K}$.

\section{Plane fluidity}

The lipid bilayer at room temperature $\left(k_{B} T / \epsilon=2.0\right)$ appears to be in a fluid phase characterized by rapid lateral diffusion of the model lipids. Typical experimental values for the in-plane diffusion coefficient in a fluid membrane are of the order of $1 \mu \mathrm{m}^{2} / \mathrm{s}^{2}$ The two-dimensional in-plane diffusion is defined as

$$
D_{x y}=\lim _{t \rightarrow \infty} \frac{\left(\Delta r_{x y}\right)^{2}}{4 t}=\lim _{t \rightarrow \infty} \frac{1}{4 N t} \sum_{i=1}^{N}\left[\left(\Delta x_{i}\right)^{2}+\left(\Delta y_{i}\right)^{2}\right],
$$

where

$$
\begin{aligned}
& \left(\Delta x_{i}\right)^{2}=\left\{\left[x_{i}(t)-x_{m}(t)\right]-\left[x_{i}(0)-x_{m}(0)\right]\right\}^{2}, \\
& \left(\Delta y_{i}\right)^{2}=\left\{\left[y_{i}(t)-y_{m}(t)\right]-\left[y_{i}(0)-y_{m}(0)\right]\right\}^{2},
\end{aligned}
$$

where $x_{m}$ and $y_{m}$ denote the coordinates of the center of mass of the system. Figure 6 shows the results of the numerical simulations at different temperatures. The diffusion coefficients, i.e., the slopes of the best linear fits to the computed data in Fig. 6, are given in Table II.

Experimental diffusion coefficients in the solid (or gel) phase, the translational diffusion coefficient ranges between $D_{x y} \sim 10^{-11}-10^{-16} \mathrm{~cm}^{2} / \mathrm{s}$, while in the fluid phase, $D_{x y}$

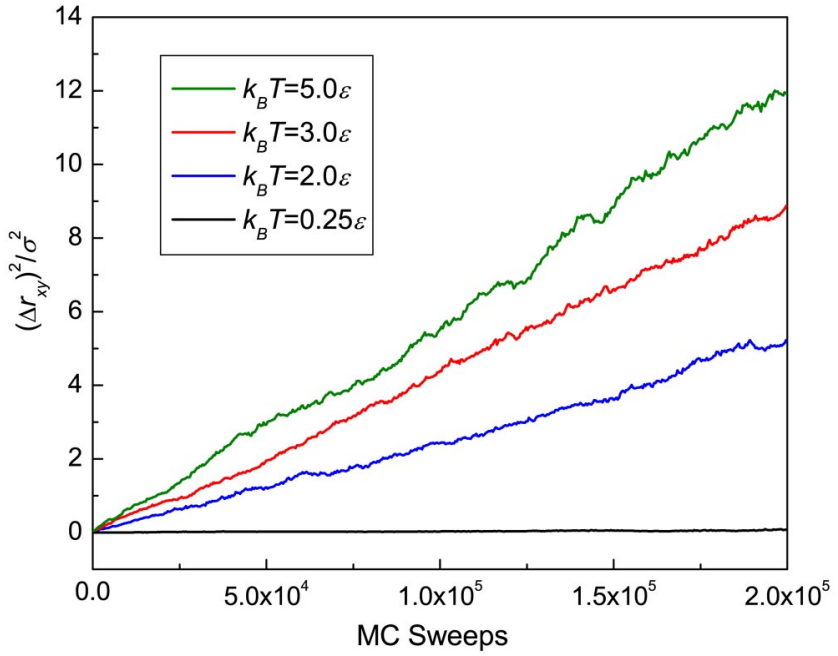

FIG. 6. Temperature dependence of the lateral diffusion of surfactant molecules in membranes at zero lateral pressure.

$\sim 10^{-8}-10^{-7} \mathrm{~cm}^{2} / \mathrm{s}^{2}$ Strictly speaking, Monte Carlo simulations do not provide dynamical information. However, if we assume that the algorithm can adequately reproduce the diffusive motion of the lipid molecules then, by using the defined length scale $\sigma \sim 5-8 \AA$, we can relate the experimental and simulated diffusion coefficients to obtain an estimate of the Monte Carlo "time." We find that, depending on the mapping, one MC sweep roughly corresponds to a time interval between $10^{-13}$ and $10^{-11} \mathrm{~s}$. In what follows, we will not consider dynamical phenomena.

Figure 7 shows the two-dimensional radial distribution function $g\left(r_{x y}\right)$. Inspection of this radial distribution function confirms that, for $T^{*}>0.8$, the bilayer is in the fluid phase.

\section{B. Elastic properties}

\section{Landau free energy}

On length scales larger than its thickness, membranes can be viewed as a continuous elastic sheet. To lowest order in the deformations, the coarse-grained (Landau) free energy of such a flexible membrane is of the following form:

$$
F=\int d A\left[\gamma+2 \kappa_{c}\left(H-c_{0}\right)^{2}+\kappa_{G} K\right],
$$

where $\gamma$ is the surface tension, $A$ is the surface area of the membrane, $\kappa_{c}$ is the bending modulus, and $\kappa_{G}$ is the saddlesplay modulus. The quantities $c_{0}, H$, and $G$ are, respectively, the spontaneous, the mean, and the Gaussian curvatures.

TABLE II. Temperature dependence of in-plane "diffusion coefficients" of surfactant molecules in a membrane at zero lateral pressure.

\begin{tabular}{cc}
\hline \hline$k_{B} T / \epsilon$ & $D_{x y}\left(10^{-5} \sigma^{2} / \mathrm{MC}\right.$ sweep $)$ \\
\hline 0.25 & $2.7201 \times 10^{-3} \pm 0.0008 \times 10^{-3}$ \\
2.0 & $2.7606 \pm 0.0007$ \\
3.0 & $4.5482 \pm 0.0005$ \\
5.0 & $6.7588 \pm 0.0014$ \\
\hline
\end{tabular}




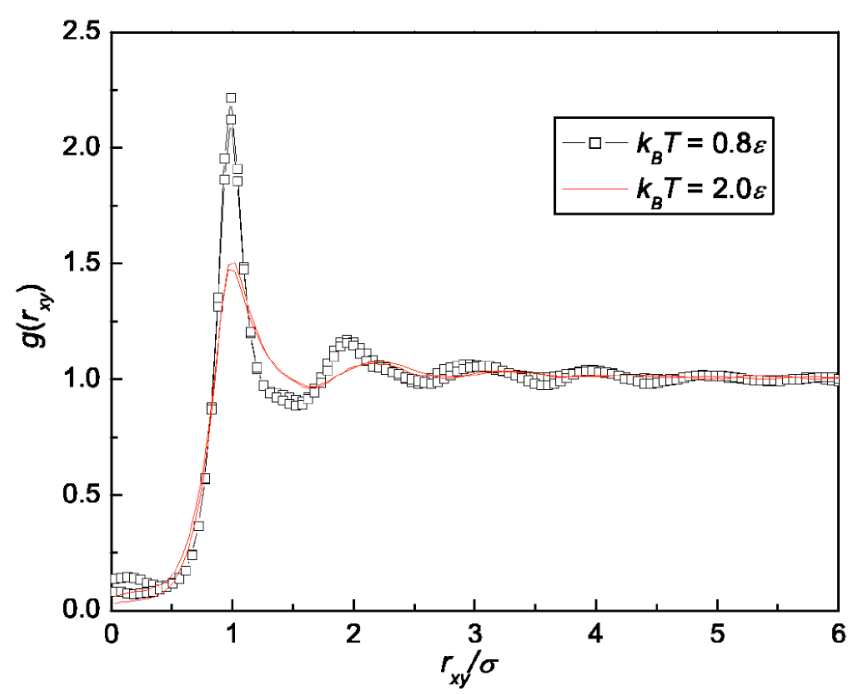

FIG. 7. In-plane radial distributions of the fluid membranes at two different temperatures. The lateral pressure, $\Sigma$, is zero.

When a tensionless membrane with area $A_{0}$ is deformed (stretched or compressed), its surface tension changes. To lowest order in the deformation:

$$
\gamma=\kappa_{A} \frac{A-A_{0}}{A_{0}},
$$

where $\kappa_{A}$ is the compressibility modulus, defined as

$$
\kappa_{A}=A_{0} \frac{\partial \gamma}{\partial A} .
$$

The change in free energy of the membrane is then given by

$$
\int \gamma d A=\kappa_{A} \frac{\left(A-A_{0}\right)^{2}}{2 A_{0}} .
$$

The total free energy of the deformed membrane is

$$
F=\kappa_{A} \frac{\left(A-A_{0}\right)^{2}}{2 A_{0}}+\int d A\left[2 \kappa_{c}\left(H-c_{0}\right)^{2}+\kappa_{G} K\right] .
$$

Using the Monge parametrization, we can write

$$
\mathbf{r}=[x, y, h(x, y)],
$$

and we have

$$
\begin{aligned}
& H=\frac{\left(1+h_{x}^{2}\right) h_{y y}+\left(1+h_{y}^{2}\right) h_{x x}-2 h_{x} h_{y} h_{x y}}{2 \sqrt{\left(1+h_{x}^{2}+h_{y}^{2}\right)^{3}}}, \\
& K=\frac{h_{x x} h_{y y}-h_{x y}^{2}}{\left(1+h_{x}^{2}+h_{y}^{2}\right)^{2}} \\
& d A=d x d y\left(1+h_{x}^{2}+h_{y}^{2}\right)^{1 / 2}
\end{aligned}
$$

For a membrane with a small curvature, $h_{x} \ll 1, h_{y} \ll 1$, the mean and Gaussian curvatures and the area can be approximately written as

$$
H \approx \frac{1}{2}\left(h_{x x}+h_{y y}\right),
$$

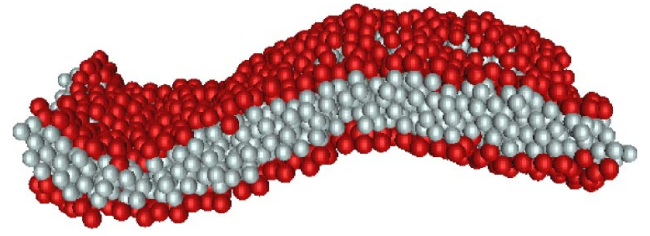

FIG. 8. A snapshot of a $N V T A_{p}$ simulation of a membrane with 2040 molecules at $k_{B} T / \epsilon=2.0$.

$$
K \approx h_{x x} h_{y y}-h_{x y}^{2},
$$

and

$$
\begin{aligned}
& d A \approx d x d y\left[1+\frac{1}{2}\left(h_{x}^{2}+h_{y}^{2}\right)\right], \\
& A \approx A_{p}+\int_{A_{p}} \frac{1}{2}\left(h_{x}^{2}+h_{y}^{2}\right),
\end{aligned}
$$

where $A_{p}$ is the projected area of the membrane in the $x-y$ plane.

In this paper, only one kind of lipid molecules is used and hence the membrane is symmetric, from which it follows that $c_{0}$ is zero. As we do not change the topology of the membrane, the saddle-splay free energy, $\int d A \kappa_{G} K$, yields a constant contribution that is included in the reference free energy, $F_{0}$. From Eqs. (21)-(24), we can express the change in free energy due to the changes in area and curvature as

$$
\begin{aligned}
F= & \kappa_{A} \frac{\left(A_{p}+1 / 2 \int_{A_{p}} d x d y|\nabla h|^{2}-A_{0}\right)^{2}}{2 A_{0}} \\
& +\frac{1}{2} \kappa_{c} \int_{A_{p}} d x d y\left(1+\frac{1}{2}|\nabla h|^{2}\right)\left|\nabla^{2} h\right|^{2} .
\end{aligned}
$$

Omitting all terms $\mathcal{O}\left(h^{3}\right)$, we have

$$
\begin{aligned}
F= & \kappa_{A} \frac{\left(A_{p}-A_{0}\right)^{2}}{2 A_{0}}+\kappa_{A} \frac{A_{p}-A_{0}}{2 A_{0}} \int_{A_{p}} d x d y|\nabla h|^{2} \\
& +\frac{1}{2} \kappa_{c} \int_{A_{p}} d x d y\left|\nabla^{2} h\right|^{2} .
\end{aligned}
$$

We can define a surface tension associated with the change in the projected surface area,

$$
\gamma_{p}=\kappa_{A} \frac{A_{p}-A_{0}}{A_{0}}
$$

then the free energy of the membrane can be written as

$$
\begin{aligned}
F= & \frac{1}{2} \gamma_{p}\left(A_{p}-A_{0}\right)+\frac{1}{2} \gamma_{p} \int_{A_{p}} d x d y|\nabla h|^{2} \\
& +\frac{1}{2} \kappa_{c} \int_{A_{p}} d x d y\left|\nabla^{2} h\right|^{2} .
\end{aligned}
$$

\section{Bending modulus}

We compute the bending elasticity of our model membranes in both $N V T A_{p}$ and NVT $\Sigma$ MC ensembles. We considered square membranes consisting of 2040 molecules. Figure 8 shows a snapshot of the membrane. 
Equation (28) is expected to be valid only on length scales where continuum theory applies. On length scales smaller than the thickness of the membranes, protrusion modes will dominate the undulatory motion of membranes and Eq. (28) breaks down. In order to use Eq. (28) to get bending elasticity, when we map the membrane on a discrete grid, we choose $N_{G}$, the number of grid points in each direction, such that mesh size $l=L / N_{G}$ is larger than the membrane's thickness $h$, where $L$ is the side length of the membranes. The local height of the membrane is defined as the mean value of the heights of the two monolayers inside a grid cell. The height of the individual monolayers is defined as the cell-averaged height of the centers of mass of the hydrophilic head groups. The discrete form of Eq. (28) is

$$
F=\frac{1}{2} \gamma_{p}\left(A_{p}-A_{0}\right)+l^{2} \sum_{\mathbf{n}}\left[\frac{1}{2} \gamma_{p}\left|\nabla h_{\mathbf{r}}\right|^{2}+\frac{1}{2} \kappa_{c}\left|\nabla^{2} h_{\mathbf{r}}\right|^{2}\right],
$$

where $\quad \mathbf{n}=n_{x} \mathbf{i}+n_{y} \mathbf{j} \quad\left(n_{x}, n_{y}=-n_{l}, \ldots,-1,0,1, \ldots, n_{r}, \quad n_{l}\right.$ $=\left[N_{G} / 2\right]$, and $\left.n_{r}=\left[\left(N_{G}-1\right) / 2\right]\right)$, and $\mathbf{r}=\mathbf{l n}$. Using a discrete Fourier transform, we have

$$
h_{\mathbf{q}}=\frac{l}{L} \sum_{\mathbf{n}} h_{\mathbf{r}} e^{i \mathbf{q} \cdot \mathbf{r}}
$$

and

$$
h_{\mathbf{r}}=\frac{l}{L} \sum_{\mathbf{q}} h_{\mathbf{q}} e^{-i \mathbf{q} \cdot \mathbf{r}},
$$

where $\mathbf{q}$ is the two-dimensional wave vector, i.e., $\mathbf{q}$ $=2 \pi \mathbf{n} / L$. In Fourier space, the Landau free energy is

$$
F=\frac{l^{2}}{2} \sum_{\mathbf{q}}\left(\gamma_{p}|\mathbf{q}|^{2}+\kappa_{c}|\mathbf{q}|^{4}\right)\left|h_{\mathbf{q}}\right|^{2}+\frac{\gamma_{p}}{2}\left(A_{p}-A_{0}\right)
$$

In the $N A_{p} T$ ensemble,

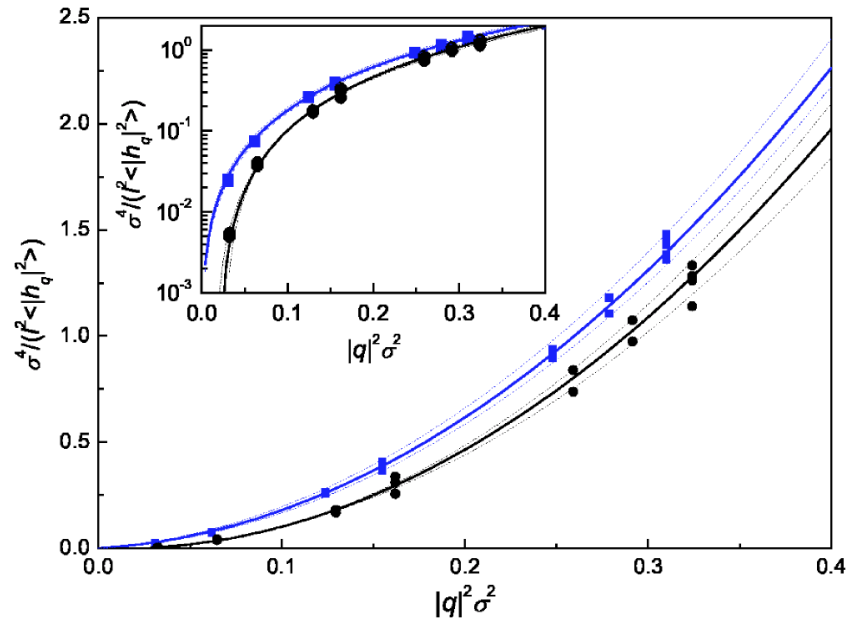

FIG. 9. Fluctuation spectrum of the membrane height at a constant projected area. The inset shows the spectrums with a vertically logarithmic coordinate. The projected areas, $A_{p}$, are, respectively, $(34.91 \sigma)^{2}$ (circle) and $(35.70 \sigma)^{2}$ (square). The drawn curves are best fits of Eq. (34) to the computed thermal fluctuations in the small wave-vector range.
TABLE III. Bending moduli of membranes at $k_{B} T / \epsilon=2.0$ from the $N V T A_{p}$ simulations. The number of the molecules in the membrane is 2040. Simulations were performed for two different membrane deformations. The computed values for $\kappa_{c}$ are very nearly the same.

\begin{tabular}{ccc}
\hline \hline$A_{p} / \sigma^{2}$ & $(34.91)^{2}$ & $(35.70)^{2}$ \\
\hline$\gamma_{p} \sigma^{2} / k_{B} T$ & $-0.3 \pm 0.1$ & $0.5 \pm 0.1$ \\
$\kappa_{c} / k_{B} T$ & $13.1 \pm 1.1$ & $12.9 \pm 0.6$ \\
$h_{c c} / \sigma$ & $3.805 \pm 0.01$ & $3.790 \pm 0.009$ \\
\hline \hline
\end{tabular}

$$
\left\langle l^{2}\left|h_{\mathbf{q}}\right|^{2}\right\rangle_{N A_{p} T}=\frac{\iint d h_{\mathbf{q}} d h_{\mathbf{q}}^{*} l^{2}\left|h_{\mathbf{q}}\right|^{2} e^{-\beta F}}{\iint d h_{\mathbf{q}} d h_{\mathbf{q}}^{*} e^{-\beta F}}=\frac{k_{B} T}{\gamma_{p}|\mathbf{q}|^{2}+\kappa_{c}|\mathbf{q}|^{4}},
$$

and

$$
\frac{1}{\left\langle l^{2}\left|h_{\mathbf{q}}\right|^{2}\right\rangle_{N A_{p} T}}=\frac{\gamma_{p}|\mathbf{q}|^{2}+\kappa_{c}|\mathbf{q}|^{4}}{k_{B} T} .
$$

In the $N \Sigma T$ ensemble,

$$
\left\langle l^{2}\left|h_{\mathbf{q}}\right|^{2}\right\rangle_{N \Sigma T}=\left\langle\frac{k_{B} T}{\gamma_{p}|2 \pi \mathbf{n} / L|^{2}+\kappa_{c}|2 \pi \mathbf{n} / L|^{4}}\right\rangle_{N \Sigma T} .
$$

When $\Sigma=0$, we can assume $\gamma_{p} \rightarrow 0$, and we have

$$
\left\langle l^{2}\left|h_{\mathbf{q}}\right|^{2}\right\rangle_{N \Sigma T(\Sigma=0)} \simeq \frac{k_{B} T\left\langle L^{4}\right\rangle_{N \Sigma T(\Sigma=0)}}{\kappa_{c}|2 \pi \mathbf{n}|^{4}} .
$$

Figure 9 shows the thermal-fluctuation spectrums of the membranes for two simulations in the $N A_{p} T$ ensemble. Equation (34) is valid only for thermal fluctuations in the small wave-vector range. Fitting these data to Eq. (34), we obtained the bending modulus of the membranes, as shown in Table III. The average head-group separation between the two membrane layers, $h_{c c}$, is also shown in Table III. Figure 10 shows the thermal-fluctuation spectrum of the membranes in the $N \Sigma T(\Sigma=0)$ ensemble. Fitting the data in Fig. 10 to Eq.

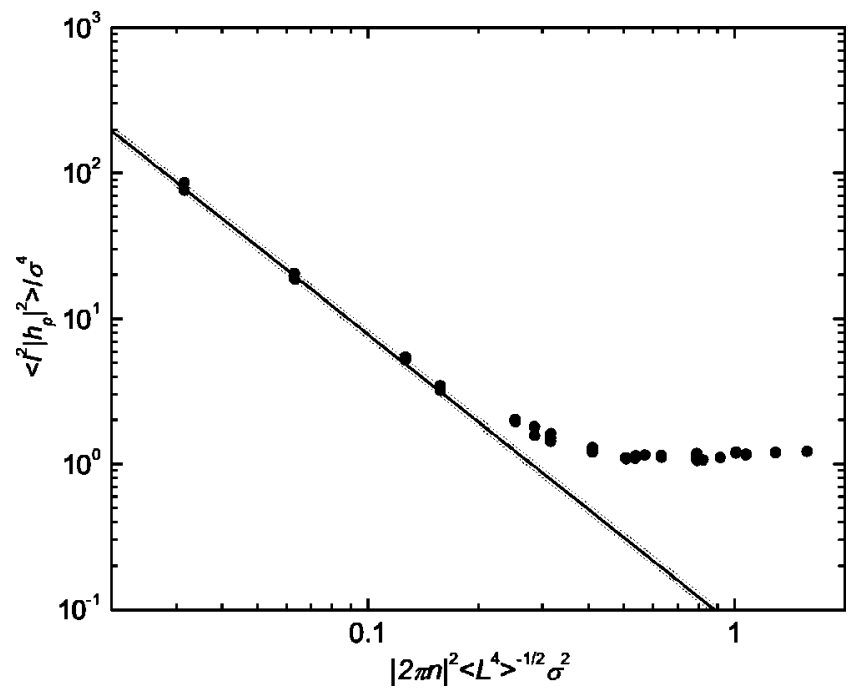

FIG. 10. Fluctuation spectrum of the membrane height at vanishing surface tension. The drawn curve is the best fit of Eq. (36) to the computed thermal fluctuations in the small wave-vector range. At large wave vectors, the fluctuation spectrum is dominated by the "white" noise due to uncorrelated protrusions. 
(36), we obtained $\kappa_{c}=12.8 \pm 1.0 k_{B} T$, which is consistent with the data from $N A_{p} T$ simulations. The bending moduli of typical biological membranes have values in the range of $10-28 k_{B} T .{ }^{34}$ Our numerical estimates for $\kappa_{c}$ fall within this range. We note that the membranes generated by the present model appear to be more flexible than those obtained in the solvent-free simulations of Refs. 26 and 27.

\section{Compressibility/stretching elasticity}

In experiments, the compressibility/stretching moduli of membranes, $\kappa_{A}$, are obtained from the measured relation between the apparent area, $A_{p}$, of vesicles and the applied tension. ${ }^{34}$ In simulations, Feller and Pastor $^{35}$ derived estimates for $\kappa_{A}$ not only from relation between tension and surface area, but also from the area fluctuations of the membranes:

$$
\kappa_{A}=\frac{k_{B} T\left\langle A_{p}\right\rangle_{N \Sigma T}}{\left\langle\delta A_{p}^{2}\right\rangle_{N \Sigma T}},
$$

where $A_{p}$ is the projected area, and $\left\langle\delta A_{p}^{2}\right\rangle_{N \Sigma T}=\left\langle A_{p}^{2}\right\rangle_{N \Sigma T}$ $-\left\langle A_{p}\right\rangle_{N \Sigma T}^{2}$. Feller and Pastor observed that the fluctuation expression yielded rather noisy data for $\kappa_{A}$. Moreover, the value for $\kappa_{A}$ thus estimated was more than four times larger than the one obtained in "mechanical" measurements. In the present paper, we calculated area compressibility/stretching elasticity of membranes through both mechanical and thermal-fluctuational measures.

Taking the values of $\gamma_{p}$ obtained from the $N V T A_{p}$ simulations (see Table III) and inserting it into Eq. (27), we find that

$$
\kappa_{A}=17.9 \pm 4.0 k_{B} T / \sigma^{2},
$$

and

$$
A_{0}=1240 \pm 30 \sigma^{2},
$$

where $\kappa_{A}$ is compressibility modulus, and $A_{0}$ is the saturated area of the membrane with 2040 molecules at $k_{B} T / \epsilon=2.0$. Typical experimental data concerning the area per molecule at zero tension range from about $0.596 \mathrm{~nm}^{2}$ for dimyristoyl phosphatidylchopine (DMPC) to $0.725 \mathrm{~nm}^{2}$ for diolcoyl phosphatidylcholine (DOPC). ${ }^{36}$ Using the length scale $\sigma$ $\sim 5-8 \AA$, the zero tension area per lipid of our simulation model is about $0.304-0.778 \mathrm{~nm}^{2}$, which covers the experimental range. Assuming that the membrane area is extensive, we can estimate $A_{0}$ for the $N=1152$ system at the same temperature to be

$$
A_{0}=701 \pm 16 \sigma^{2} \text {. }
$$

In addition, we performed NVT $\Sigma \mathrm{MC}$ simulations to estimate $\kappa_{A}$. The number of molecules in the simulated membranes was chosen to be 1152 . We performed a rather long simulation (300 $000 \mathrm{MC}$ cycles) to equilibrate the membrane for a given value of the applied tension. The temporal evolution of the projected surface area of the membrane in equilibrium is shown in Fig. 11. Large fluctuations are observed in all cases. More importantly, the time scale of the fluctuations is not short compared to the length of the simulations. Under these circumstances, Eq. (37) is not expected to yield reliable

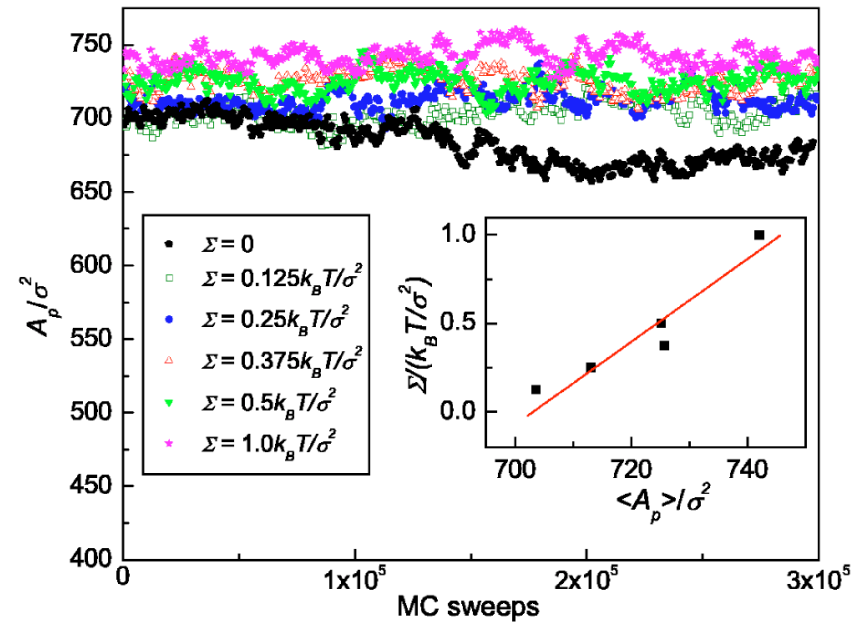

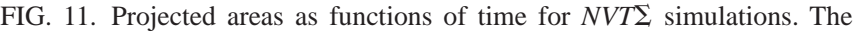
inset shows the profile of lateral pressure vs statistical projected area, where the line gives the best linear fit to the computed data.

results as we are likely to underestimate the magnitude of the fluctuations. Table IV lists our estimates for the average projected areas, root-mean-squared (rms) fluctuations in $A_{p}$, and the corresponding compressibility moduli obtained by using Eq. (37) for each of the $\Sigma>0$ simulations. These computations lead to an average value of compressibility moduli as $\kappa_{A}=13 \pm 2 k_{B} T / \sigma^{2}$, which is somewhat smaller than the value in Eq. (38). The slope $\partial \Sigma / \partial A_{p}$ is also obtained by roughly fitting the lateral stress as a linear function of the projected area in Fig. 11, which leads to $\partial \Sigma / \partial A_{p}=0.023 \pm 0.004 k_{B} T$. We did not compute the lateral pressure profile in our simulations. Although this is feasible in principle, the presence of many-body forces in our model makes this nontrivial. However, since membranes in quite long MC simulations are expected to be in force equilibrium, we can assume $\Sigma \approx \gamma_{p}$. According to Eq. (27), we have

$$
\frac{\partial \Sigma}{\partial A_{p}} \approx \frac{\kappa_{A}}{A_{0}} .
$$

Using the value of $A_{0}$ in Eq. (40), we then obtain $\kappa_{A}$ $=16 \pm 3 k_{B} T / \sigma^{2}$. We note that the different estimates for $\kappa_{A}$, though noisy, are not mutually inconsistent.

In experiments, $\kappa_{A} \sim 229-265 \mathrm{mN} / \mathrm{m}$ at room temperature. ${ }^{34}$ Using the length scale $\sigma \sim 5-8 \AA$, the typical experimental values for $\kappa_{A}$ are $14-40 k_{B} T / \sigma^{2}$ at room temperature. $\kappa_{A}$ of our simulations roughly lies in the experimental range.

TABLE IV. Mean values and rms fluctuations for the projected area calcu-

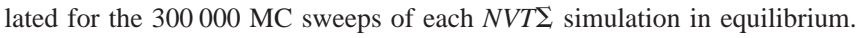
Each of the simulated membranes has 1152 molecules. The values of the compressibility/stretching modulus were calculated by using Eq. (37).

\begin{tabular}{llll}
\hline \hline$\Sigma /\left(k_{B} T / \sigma^{2}\right)$ & $\left\langle A_{p}\right\rangle / \sigma^{2}$ & $\left\langle\delta A_{p}{ }^{2}\right\rangle / \sigma^{4}$ & $\kappa_{A} /\left(k_{B} T / \sigma^{2}\right)$ \\
\hline 0.125 & 703.75 & 64.02465 & 10.99 \\
0.25 & 713.10 & 50.74 & 14.05 \\
0.375 & 725.68 & 57.30 & 12.67 \\
0.5 & 725.12 & 53.31 & 13.60 \\
1.0 & 742.07 & 48.55 & 15.29 \\
\hline
\end{tabular}




\section{Elastic ratio}

Evans and Rawicz proposed that $\kappa_{c} \sim \kappa_{A} h^{2}$, where $h$ is the membrane thickness. ${ }^{37}$ This qualitative relation between $\kappa_{c}$ and $\kappa_{A}$ was also obtained by a scaling analysis based on the molecular theory of chain packing. ${ }^{2}$ The elastic ratio is defined as $b=\kappa_{A} h^{2} / \kappa_{c}$. The value of $b$ is model-dependent. For instance, for a polymer-brush bilayer, where two monolayers are held together by hydrophobic interactions, the theoretical analysis of Ref. 34 predicts $b=24$. For an unbounded isotropic-elastic bilayer, where the two monolayers can slide freely against each other, ${ }^{34,38}$ the expected value of $b$ is $b$ $=48$, and for a pair of isotropic layers that cannot slide with respect to each other $b=12 .{ }^{34}$

Experimental data on membrane elasticity indicate that typical values of the elastic ratio, $b$, are $20-30 .^{34}$ An early solvent-explicit CG molecular simulation gave $b \approx 48 .{ }^{38} \mathrm{~A}$ recent molecular simulation yielded $b \approx 24{ }^{16}$

In our model, the thickness of the membrane $h$ is determined by the packing of hydrocarbon chains. It is therefore reasonable to identify $h$ with the distance $h_{c c}$ between hydrocarbon head groups. From the data in Secs. III B 2 and III B 3, we obtain $b=15-25$ for the simulated membranes, which is, again in the typical experimental range. Comparison between our numerical results and the theoretical analysis of Ref. 34 suggests that our model membranes behave not unlike polymer-brush bilayers.

\section{CONCLUSIONS}

This paper explores the properties of a simple, solventfree coarse-grained model for flexible bilayer membranes. In the model, the amphiphilic molecules are described as flexible three-bead chains, and the solvent molecules are eliminated by compensating the interaction between the solvent and amphiphilic molecules with a multibody potential which depends on the density of hydrophobic tail beads.

For the modeled membrane, the solid phase only exists at low temperature $\left(k_{B} T / \epsilon<0.5\right)$. At higher temperatures, the bilayer membrane is in a two-dimensional (2D) liquid state. Comparison of the simulated lateral diffusion coefficient of fluid bilayer membranes with the corresponding experimental data allows us to identify an effective time interval to a single MC cycle.

From the thermal fluctuation spectrum of membranes with constant projected areas and from the lateral stressstrain relation, we have extracted the bending modulus, the area stretching/compressibility modulus, and the elastic ratio between them. In spite of its great simplicity, the present solvent-free coarse-grained model yields a surprisingly good description of the elastic properties of fluid membranes, as studied in the experiments and in fully atomistic simulations.
In future work, we aim to study the properties of defects in these membranes.

\section{ACKNOWLEDGMENTS}

The work of the FOM Institute is part of the research program of the Stichting voor Fundamenteel Onderzoek der Materie (FOM), which is financially supported by the Nederlandse Organisatie voor Wetenschappelijk Onderzoek (NWO). Computer time at SARA is also gratefully acknowledged.

${ }^{1}$ B. Alberts, A. Johnson, J. Levis et al., Molecular Biology of the Cell, 4th ed. (Garland Science, New York, 2002).

${ }^{2}$ R. Lipowsky and E. Sackmann, Structure and Dynamics of Membranes from Cells to Vesicles (Elsevier Science, Amsterdam, 1995).

${ }^{3}$ C. Hofsab, E. Lindahl, and O. Edholm, Biophys. J. 84, 2192 (2003).

${ }^{4}$ D. P. Tieleman, H. Leontiadou, A. E. Mark, and S.-J. Marrink, J. Am. Chem. Soc. 125, 6382 (2003).

${ }^{5}$ Y. Kantor, M. Kardar, and D. R. Nelson, Phys. Rev. A 35, 3056 (1987).

${ }^{6}$ F. F. Abraham, W. Rudge, and M. Plischke, Phys. Rev. Lett. 62, 1757 (1989).

${ }^{7}$ R. Lipowsky and B. Zielenska, Phys. Rev. Lett. 62, 1572 (1989).

${ }^{8}$ A. Baumgartner and J.-S. Ho, Phys. Rev. A 41, 5747 (1990).

${ }^{9}$ R. Lipowsky and M. Girardet, Phys. Rev. Lett. 65, 2893 (1990).

${ }^{10}$ F. L. H. Brown, Biophys. J. 84, 842 (2003).

${ }^{11}$ L. C.-L. Lin and F. L. H. Brown, Phys. Rev. Lett. 93, 256001 (2004).

${ }^{12}$ J. Shillcock and R. Lipowsky, J. Chem. Phys. 117, 5048 (2002).

${ }^{13}$ C. Stadler, H. Lange, and F. Schmid, Phys. Rev. E 59, 4248 (1999).

${ }^{14}$ F. M. Haas and R. Hilfer, J. Chem. Phys. 105, 3859 (1996).

${ }^{15}$ S. Yamamoto, Y. Maruyama, and S.-A. Hyodo, J. Chem. Phys. 116, 5842 (2002).

${ }^{16}$ E. Lindahl and O. Edholm, Biophys. J. 79, 426 (2000).

${ }^{17}$ W. K. den Otter and W. J. Briels, J. Chem. Phys. 118, 4712 (2003).

${ }^{18}$ M. Kranenburg, M. Venturoli, and B. Smit, Phys. Rev. E 67, 060901(R) (2003).

${ }^{19}$ R. D. Groot and K. L. Rabone, Biophys. J. 81, 725 (2001).

${ }^{20}$ T. Soddemann, B. Dunweb, and K. Kremer, Eur. Phys. J. E 6, 409 (2001).

${ }^{21}$ G. Ayton, S. G. Bardenhagen, P. McMurty, D. Sulsky, and G. A. Voth, J. Chem. Phys. 114, 6913 (2001).

${ }^{22}$ F. M. Haas, P.-Y. Lai, and K. Binder, Makromol. Chem., Theory Simul. 2, 889 (1993)

${ }^{23}$ M. Muller and M. Schick, J. Chem. Phys. 105, 8282 (1996).

${ }^{24}$ J.-M. Drouffe, A. C. Maggs, and S. Leibler, Science 254, 1353 (1991).

${ }^{25}$ H. Noguchi and M. Takasu, J. Chem. Phys. 115, 9547 (2001).

${ }^{26}$ O. Farago, J. Chem. Phys. 119, 596 (2003).

${ }^{27}$ G. Brannigan and F. L. H. Brown, J. Chem. Phys. 120, 1059 (2004).

${ }^{28}$ G. Brannigan, A. C. Tamboli, and F. L. H. Brown, J. Chem. Phys. 121, 3259 (2004)

${ }^{29}$ W. Helfrich, Z. Naturforsch. A 28C, 693 (1973).

${ }^{30}$ N. Dan, P. Pincus, and S. A. Safran, Langmuir 9,2768 (1993).

${ }^{31}$ R. Golestanian, M. Goulian, and M. Kardar, Phys. Rev. E 54, 6725 (1996).

${ }^{32}$ R. Menes and S. A. Safran, Phys. Rev. E 56, 1891 (1997).

${ }^{33}$ K. S. Kim, J. Neu, and G. Oster, Phys. Rev. E 61, 4281 (2000).

${ }^{34}$ W. Rawicz, K. C. Olbrich, T. Mcintosh, D. Needham, and E. Evans, Biophys. J. 79, 328 (2000).

${ }^{35}$ S. E. Feller and R. W. Pastor, J. Chem. Phys. 111, 1281 (1999).

${ }^{36}$ J. F. Nagle and S. Tristram-Nagle, Biochim. Biophys. Acta 1469, 159 (2000).

${ }^{37}$ E. Evans and W. Rawicz, Phys. Rev. Lett. 64, 2094 (1990).

${ }^{38}$ R. Goetz, G. Gompper, and R. Lipowsky, Phys. Rev. Lett. 82, 221 (1999). 